\title{
Ultrasound-targeted microbubble destruction enhances polyethylenimine-mediated gene transfection in vitro in human retinal pigment epithelial cells and in vivo in rat retina
}

\author{
CAIFENG WAN $^{1}$, JIN QIAN ${ }^{2}$, FENGHUA LI $^{1}$ and HONGLI LI ${ }^{1}$ \\ ${ }^{1}$ Department of Ultrasound, Renji Hospital, School of Medicine, Shanghai Jiao Tong University, Shanghai 200127; \\ ${ }^{2}$ Department of Ophthalmology, The Baoshan Branch Institute of Shanghai Shuguang Hospital, Shanghai 201900, P.R. China
}

Received June 26, 2014; Accepted March 16, 2015

DOI: $10.3892 / \mathrm{mmr} .2015 .3703$

\begin{abstract}
The aim of the present study was to investigate the efficacy and safety of ultrasound-targeted microbubble destruction (UTMD)-mediated polyethylenimine (PEI) transfection in cultured human retinal pigment epithelial (RPE) cells in vitro and rat retinas in vivo. An enhanced green fluorescent protein plasmid (pEGFP) was incubated with PEI to prepare a cationic complex (PEI/pEGFP), which was confirmed using a gel retardation assay. In the in vitro study, cultured human RPE cells were subjected to US waves under different conditions with or without microbubbles. The effect of UTMD on the viability of the cells was evaluated. In the in vivo study, gene transfer was examined by injecting PEI/pEGFP into the subretinal space of the rats. The rats treated with PEI/pEGFP and UTMD served as the experimental group, while rats treated with PEI/pEGFP alone served as the control group. The transfected tissue was visualized using an inverted fluorescence microscope. The expression of EGFP was classified into three groups, negative, weak positive and strong positive. Hematoxylin and eosin staining of frozen sections was used to observe tissue damage and the location of the EGFP gene expression. The electrophoresis experiment revealed that PEI treatment was able to condense DNA efficiently. In the in vitro study, the gene transfer efficiency under the optimal UTMD condition was enhanced and significantly higher than control groups. In the in vivo study, UTMD was able to enhance transgene expression in the retina without marked tissue damage. Frozen sections of the optic cups exhibited pEGFP-positive cells, predominantly distributed in the retina. This noninvasive
\end{abstract}

Correspondence to: Mrs. Fenghua $\mathrm{Li}$ and Mrs. Hongli Li, Department of Ultrasound, Renji Hospital, School of Medicine, Shanghai Jiao Tong University, 1630 Dongfang Road, Shanghai 200127, P.R. China

E-mail: lifenghuaprof@sina.com

E-mail: lihonglidoctor@sina.com

Key words: ultrasound, microbubbles, polyethylenimine, retinal pigment epithelial cells, retina novel combination of UTMD with PEI was able to enhance targeted gene delivery and gene expression in the rat retina without causing any apparent tissue damage, and may be a safe method to transfer genes and drug treatments directly to the retina, therefore being of potential therapeutic value.

\section{Introduction}

Millions of individuals suffer from inherited and acquired retinal diseases, which have become the leading cause of blindness in adults $(1,2)$. However, no satisfactory treatment is currently available for these disorders. As insight into the molecular mechanisms of the ocular diseases has increased, gene therapy has been proposed as a promising therapeutic tool for the treatment of ocular diseases. Successful gene therapy depends on efficient gene transfer to the targeted cells to provide stable gene expression with minimal toxicity. How to render foreign gene expression safe and efficient in target cells is a key issue. Although the use of a viral vector is able to provide a high transfer efficiency, the limited payload capacity, difficult large scale production and safety issues, such as potential immunogenicity and oncogenesis may hamper their clinical application (3-5). Nonviral vectors have attracted interest, as they are simple to prepare, stable, easy to modify and safe compared with viral vectors. However, they are also disadvantaged by a low transfection efficiency and transient gene expression $(6,7)$. These limitations have prompted the requirement for the development of an effective delivery method, which has a high level of biosafety and a low level of cytotoxicity.

Ultrasound (US)-targeted microbubble (MB) destruction (UTMD)-mediated gene delivery systems hold promise as effective delivery methods (8-12). Numerous proof-of-principle studies have indicated that ultrasonic irradiation itself promotes gene transfection and expression, UTMD is able to further enhance the gene transfection efficiency in vitro and in vivo (8-10). It has become an intense area of investigation as it is a non-invasive, target-specific type of gene delivery. In the sphere of ophthalmology, the application of UTMD-mediated gene therapy has been observed to be efficient in previous studies $(11,12)$.

Polyethylenimine (PEI) is a cationic polymer with a high charge density, which has been widely examined in 
numerous studies as a gene-delivery vector (13). PEI-mediated gene delivery is based on the electrostatic interaction of the polycation with the negatively charged phosphate groups of DNA. PEI is able to condense DNA into compact particles via the electrostatic interaction with condensing compounds, protecting the DNA from degradation by nucleases or other enzymes, and the compact particles may be engulfed by cells via natural processes, such as endocytosis, pinocytosis and phagocytosis (14). The proton-sponge effect of PEI is responsible for efficient gene transfer, which is able to evade lysosomal degradation by rupture of the endosomal vesicle prior to fusion. To the best of our knowledge, UTMD-mediated PEI/enhanced green fluorescent protein plasmid (pEGFP) transfection of retinal pigment epithelial (RPE) cells in vitro and rat retina in vivo has not been previously reported. The aim of the present study was to evaluate whether the combination of UTMD and PEI was a useful and suitable tool for the transfection of RPE cells in cell culture and in vivo in the rat.

\section{Materials and methods}

pEGFP and MBs. An expression vector for the EGFP gene, pEGFP-N1 (4.7 kb), was provided by the Experimental Research Center of Shanghai Jiao Tong University Affiliated Renji Hospital (Shanghai, China). The pEGFP-N1 was purified from a culture of Escherichia coli DH5a using the EndoFree Plasmid Maxi kit (Qiagen Inc., Valencia, CA, USA) according to the manufacturer's instructions. The purity of the plasmid DNA was determined by measuring absorption at a $260 \mathrm{~nm}$ wavelength (A260) using spectrophotometry (DU-640; Bechman Coulter, Fullerton, CA, USA). The concentration of isolated plasmid DNA was resuspended to a final concentration of $1 \mu \mathrm{g} / \mu \mathrm{l}$ in buffer (Sigma-Aldrich, St. Louis, MO, USA) and stored at $-20^{\circ} \mathrm{C}$. The A260:A280 ratio of pEGFP-N1 was between 1.8 and 2.0, indicating that the purified plasmid DNA was free of contaminants.

Second generation MBs, termed SonoVue, which are coated with a thin lipid monolayer membrane shell, and consist of a gas core of $\mathrm{SF}_{6}$ were purchased from Bracco (Milan, Italy). The average diameter of the MB was between 2.5 and $6.0 \mu \mathrm{m}$. They were reconstituted in a saline solution according to the manufacturer's instructions and yielded a preparation containing $2-5 \times 10^{8} \mathrm{MBs} / \mathrm{ml}$. In order to calculate the MB cell ratio, $2 \times 10^{8} \mathrm{MBs} / \mathrm{ml}$ was used as a basis.

\section{In vitro study}

Cell culture. The ARPE-19 human RPE cell line was obtained from the American Type Culture Collection (CRL-2302; American Type Culture Collection, Rockville, MD, USA) and incubated in Dulbecco's modified Eagle's medium (DMEM; Gibco-BRL, Grand Island, NY, USA) with $10 \%$ fetal bovine serum (Gibco-BRL), and $100 \mathrm{U} / \mathrm{ml}$ penicillin and $100 \mu \mathrm{g} / \mathrm{ml}$ streptomycin, at $37^{\circ} \mathrm{C}$ in a humidified environment of $5 \% \mathrm{CO}_{2}$. The total cell count was determined using a cell counting chamber (Huarui Medical Devices Co. Ltd., Jiangsu, China). The initial cell viability was determined by means of exclusion with Trypan blue dye (Sigma-Aldrich, St. Louis, MO, USA). Dead cells were colored pale blue, whereas living cells remain unstained. Cell viability $(\%)=$ number living cells $/$ total number of living and dead cells x 100.
Preparation of transfection complex. Branched PEI with an average molecular weight of $25 \mathrm{kDa}$ was purchased from Sigma-Aldrich and diluted $1 \mathrm{mg}$ PEI coupling medium (Tianrun Medical Devices Co., Ltd., Jiangsu, China) in $1,000 \mathrm{ml}$ deionized water, neutralized with $\mathrm{HCl}$ and filtering at $0.2 \mu \mathrm{m}$ (Millipore, Bedford, MA, USA). The PEI/pEGFP complexes were developed by mixing PEI and the plasmids at $0.5: 5$ of PEI nitrogen/DNA phosphate ratio (N/P ratio). The $\mathrm{N} / \mathrm{P}$ ratio was based on the recognition that $1 \mu \mathrm{l} \mathrm{PEI}$ stock solution contained $10 \mathrm{nmol}$ amine nitrogen and $1 \mu \mathrm{g}$ DNA contained $3 \mathrm{nmol}$ phosphate (15). Agarose gel electrophoresis was performed at $80 \mathrm{~V}$ for $40 \mathrm{~min}$ to determine the $\mathrm{N} / \mathrm{P}$ ratio at which $\mathrm{PEI}$ was able to condense the DNA efficiently. The PEI/pEGFP complexes were incubated for $30 \mathrm{~min}$ at room temperature prior to the experiment. Once the cells grew to $70-80 \%$ confluence, they were transfected with the PEI/pEGFP complexes. These cells were infected with PEI/pEGFP alone or in combination with US or UTMD. The cells infected with PEI/pEGFP and UTMD served as the experimental group.

US exposure. A therapeutic US machine (Topteam 161; Chattanooga Medical Supply, Inc., Chattanooga, TN, USA) was used in the present experiment. The cells were exposed to US [frequency, $1 \mathrm{MHz}$; intensity, 1, 2 and $3 \mathrm{w} / \mathrm{cm}^{2}$; duration, 1,2 and $3 \mathrm{~min}$; pulse wave with a 20 and $50 \%$ duty cycle (DC) and continuous wave; and pulse recurrent frequency, $100 \mathrm{~Hz}$ ] with or without MB. The dosage of SonoVue was selected according to the ratio of MBs to cells (20:1, 50:1 and 80:1). The probe was placed at the bottom of the plates with a small quantity of coupling medium between them. A self-made plastic disc of the same size as the wells was placed between the probe and the bottom of the plates to ensure the same thickness of coupling medium between the probe and the plates, and to avoid ultrasonic radiation affecting the adjacent wells. In the process of the irradiation, the plate was moved slowly around the circumference of the plastic dish.

Effect of UTMD on cell viability. The viability of the ARPE-19 cells under the effect of the US with contrast agent was assessed with a cell counting kit-8 (Qiagen, Crawley, UK) according to the manufacturer's instructions. The ARPE-19 cells were transferred into the 96-well plates, in every other four wells, at a concentration of $1 \times 10^{4}$ cells/well and grown in a humidified incubator at $37^{\circ} \mathrm{C}$ and $5 \% \mathrm{CO}_{2}$ for $24 \mathrm{~h}$. When the cells reached $70-80 \%$ confluence, the medium in each well was replaced with $100 \mu \mathrm{l}$ fresh DMEM for the control group, or with a $100 \mu \mathrm{l}$ mixed solution of MBs and fresh DMEM for the UTMD group. The optimum parameters of UTMD attained from the experiment investigating cell viability were used in the following experiment.

Assessment of transfection efficiency. After the gene transfer treatment, ARPE-19 cells were incubated for $48 \mathrm{~h}$. EGFP expression was observed and images were captured using an inverted fluorescence microscope (Axio Observer Z1; Carl Zeiss, Inc., Oberkochen, Germany). The ratio of EGFP-positive RPE cells was quantitatively examined using flow cytometric analysis (Epics XL; Beckman Coulter, Miami, FL, USA). 
Table I. Distribution of the number of rats in different EGFP expression groups.

\begin{tabular}{lccc}
\hline & \multicolumn{3}{c}{ EGFP expression (n) } \\
\cline { 2 - 4 } Condition & Negative & Weak positive & Strong positive \\
\hline PEI/pEGFP & 3 & 9 & 0 \\
PEI/pEGFP+UTMD & 0 & 5 & 7 \\
\hline
\end{tabular}

pEGFP, enhanced green fluorescent protein plasmid; PEI, polyethylenimine; US, ultrasound; UTMD, ultrasound-targeted microbubble destruction.

In vivo study. Normal adult Sprague-Dawley (SD) rats (female; weight, $250 \mathrm{~g}$ ) were used in the present experiment. All animals were handled humanely in accordance with the policies stated in the Vision and Ophthalmology Statement for the Use of Animals in Ophthalmic and Vision Research and with the guidelines approved by national and local institutions. The rats were anesthetized with an intraperitoneal injection of $10 \%$ chloral hydrate. Pupillary dilatation was achieved using tropicamide eye drops (Xingqi Parmaceutical Co., Ltd., Shengyang, China). The eyes were gently protruded using a plastic circle and subsequently covered with ofloxacin eye ointment (Xingqi Parmaceutical Co., Ltd.). A 26-gauge needle was inserted $1 \mathrm{~mm}$ posterior to the corneal limbus, causing a self-sealing wound tunnel, which was observed under a surgical microscope (SM-2000 J; Eder, Shanghai, China). PEI/pEGFP complexes $(4 \mu \mathrm{l})$ alone or in combination with MBs $(1 \mu \mathrm{l})$ or normal saline $(1 \mu \mathrm{l})$ were injected into the eyes of the rats.

A total of 32 rats were used in the present study as follows: i) PEI/pEGFP group (16 rats): PEI/pEGFP complexes and normal saline, without MBs and US exposure; and ii) PEI/ pEGFP + UTMD group (16 rats): PEI/pEGFP complexes and MBs with US exposure.

Considering the risk of volume overload, the infusions were administered slowly. Directly after the subretinal injection, the eyes of the rats in the PEI/pEGFP + UTMD group were exposed to US. The frequency used was $1 \mathrm{MHz}$, US intensity was $2 \mathrm{w} / \mathrm{cm}^{2}$ and the pulse repetition frequency was $100 \mathrm{~Hz}$, with $50 \%$ DC. The entire treatment lasted for $5 \mathrm{~min}$.

A total of 12 eyes were enucleated following sacrifice via an overdose administered on day 5 after subretinal injection from each group. The fundus oculi were prepared following enucleation of the globe via removal of the anterior segment with a blade and carefully transferring the whole fundus oculi to a microscope slide. The transfected tissue was visualized using an inverted fluorescence microscope (Zeiss Axiovert S100; Zeiss, Oberkochen, Germany). The gene-transfer efficiency was observed and images were captured. If no EGFP-positive cells were observed, the cells were defined as negative; star-like EGFP-positive cells observed were defined as a weak positive; and diffuse EGFP-positive cells observed were defined as a strong positive. Two eyes were harvested from each group to produce frozen sections on day 5 . The enucleated eyes were embedded in optimal cutting temperature compound (Sakura; Torrance, CA, USA) and cryosections $(10 \mathrm{~mm})$ were obtained. Two eyes were also harvested from each group for histology on day 5 . The sections were stained with hematoxylin and eosin

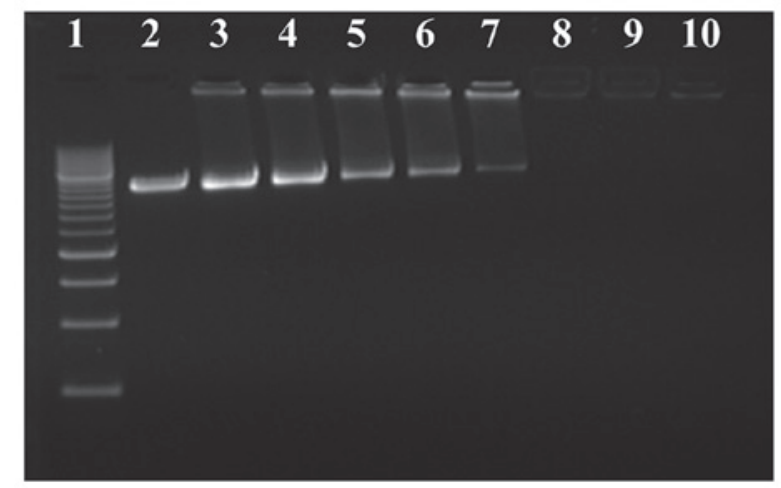

Figure 1. Electrophoretic patterns of plasmid DNA complexes prepared with PEI at various N/P ratios. Lane 1 , DNA maker; 2 , plasmid DNA alone; $3, \mathrm{~N} / \mathrm{P}=0.5 ; 4, \mathrm{~N} / \mathrm{P}=1.0 ; 5, \mathrm{~N} / \mathrm{P}=1.5 ; 6, \mathrm{~N} / \mathrm{P}=2 ; 7, \mathrm{~N} / \mathrm{P}=2.5 ; 8, \mathrm{~N} / \mathrm{P}=3 ; 9, \mathrm{~N} / \mathrm{P}=4$; and $10, \mathrm{~N} / \mathrm{P}=5$. When $\mathrm{N} / \mathrm{P} \geq 3$, the plasmid DNA migration could not be observed, and PEI was able to effectively condensate the plasmid DNA. PEI, polyethylenimine; N/P ratio, PEI nitrogen/DNA phosphate ratio.

to visualize the retinal architecture using microscopy. All specimens were assessed by two observers who were blinded to the experimental conditions and received no information regarding the nature of the specimens.

Statistical analysis. Statistical analyses were performed using the SPSS 13.0 software package (SPSS, Inc., Chicago, IL, USA). All data are expressed as the mean \pm standard deviation. All transfection conditions were performed in three parallel wells following an identical procedure and repeated three times. Multiple group comparisons were performed using a one-way analysis of variance. $\chi^{2}$ tests were used for count data analysis. $\mathrm{P}<0.05$ was considered to indicate a statistically significant difference.

\section{Results}

Gel retardation analysis of PEI/pEGFP complex. Agarose gel electrophoresis retardation analysis revealed that PEI could condense DNA efficiently (Fig. 1). With the increment of the $\mathrm{N} / \mathrm{P}$ ratio, the plasmid DNA migrated more slowly. At N/P $\geq 3$, the plasmid DNA migration could not be observed, and PEI could effectively condense the plasmid DNA. According to the results of agarose gel electrophoresis, the N/P ratio was selected as 8 in the present study.

Effect of UTMD on cell viability. The effects of UTMD on the viability of ARPE-19 cells are shown in Fig. 2. The 
A

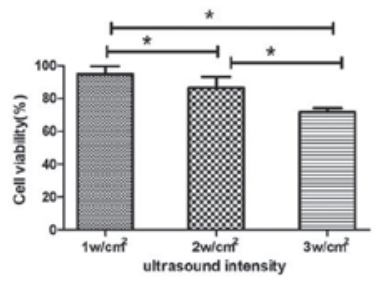

B

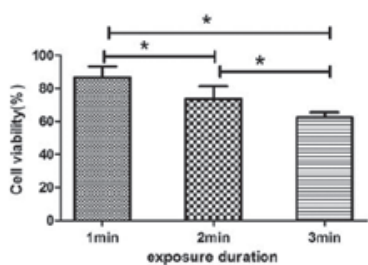

C

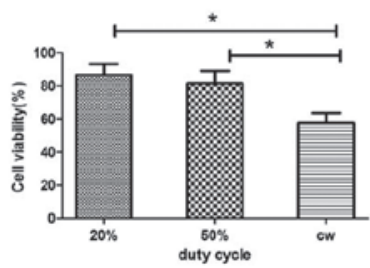

D

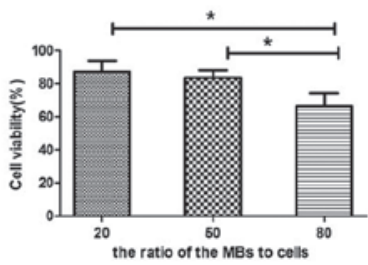

Figure 2. Effects of UTMD on the viability of ARPE-19 cells. (A) Under the condition of $3 \mathrm{w} / \mathrm{cm}^{2}$, the viability of cells was significantly lower than that in the 1 and $2 \mathrm{w} / \mathrm{cm}^{2}$ groups ( $\mathrm{P}=0.000$ and 0.000 , respectively). Cell viability under $2 \mathrm{w} / \mathrm{cm}^{2}$ was significantly lower than that under the condition of $1 \mathrm{w} / \mathrm{cm}^{2}$ $(\mathrm{P}=0.031)$. (B) Cell damage under the condition for 3 min was significantly lower than that under the condition for 1 and 2 min $(\mathrm{P}=0.004$ and 0.037 , respectively). Cell viability under 2 min was significantly lower than that under the condition of $1 \mathrm{~min}(\mathrm{P}=0.016)$. (C) Cell viability in the $20 \% \mathrm{DC}$ and $50 \% \mathrm{DC}$ group were significantly higher than that in the continuous wave group ( $\mathrm{P}=0.004$ and 0.004 , respectively). No significant difference was found between the $20 \%$ DC and 50\% DC groups. (D) Cell viability of the 80:1 group was lower than that in the 20:1 and 50:1 groups (P=0.006 and 0.006, respectively) No significant difference was found between the 20:1 and the 50:1 MBs concentration groups. US, ultrasound; UTMD, ultrasound-targeted microbubble destruction; DC, duty cycle; MBs, microbubbles.

A

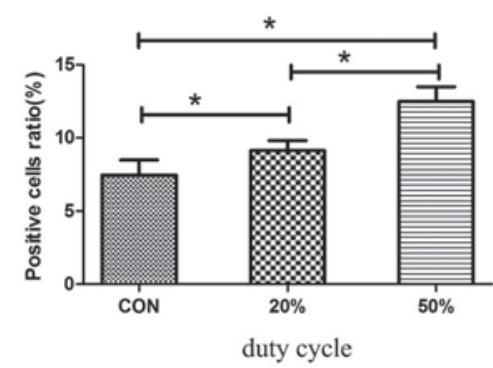

B

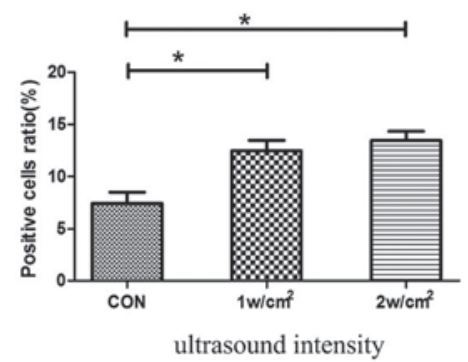

C

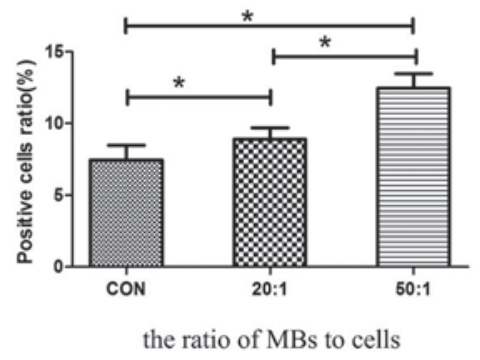

Figure 3. UTMD-mediated PEI/pEGFP transfection of ARPE-19 cells. (A) Under the conditions of a 50\% duty cycle, UTMD was able to significantly enhance transfection efficiency compared with that in the control and $20 \%$ duty cycle groups ( $\mathrm{P}=0.0001$ and 0.0001 , respectively). (B) Under the conditions of a 1 and $2 \mathrm{w} / \mathrm{cm}^{2}$, UTMD was able to significantly enhance transfection efficiency compared with that in the control group ( $\mathrm{P}=0.001$ and 0.001 , respectively). No significant difference was identified between the 1 and $2 \mathrm{w} / \mathrm{cm}^{2}$ groups. (C) Gene transfer efficiency in the 50:1 (MBs:cells) group was significantly higher than that in the 20:1 (MBs:cells) group ( $\mathrm{P}=0.0001)$, and the two were significantly higher than that in the control group ( $\mathrm{P}=0.0001$ and 0.001 , respectively). pEGFP, enhanced green fluorescent protein plasmid; PEI, polyethylenimine; US, ultrasound; UTMD, ultrasound-targeted microbubble destruction; MBs, microbubbles.

cell viability of all experimental groups is lower than that of the control group. When the DC was set at $20 \%$ and the ratio of MBs to cells set at 20:1, the increase in US intensity (between 1 and $3 \mathrm{w} / \mathrm{cm}^{2}$ ) and duration (between 1 and $3 \mathrm{~min}$ ) resulted in a decrease in cell viability (Fig. 2A and B). The cell viability was the lowest in the $3 \mathrm{w} / \mathrm{cm}^{2}(71.70 \pm 2.77 \%)$ and the 3 min groups $(62.71 \pm 2.74 \%)$, the two exhibiting levels of $<80 \%$. The cell viability in the 2 and $3 \mathrm{w} / \mathrm{cm}^{2}$ groups was significantly lower than that in the $1 \mathrm{w} / \mathrm{cm}^{2}$ group $(\mathrm{P}=0.031$ and 0.000 , respectively; Fig. 2A). The cell viability in the 2 and 3 min groups was significantly lower than that in the 1 min group $(\mathrm{P}=0.016$ and 0.004 , respectively; Fig. $2 \mathrm{~B})$. The cell viability in the $20 \%$ DC and $50 \%$ DC groups was significantly higher than that in the continuous wave group $(51.25 \pm 5.43 \%)$, but no significant difference was identified between the 20 and $50 \%$ DC groups $(86.67 \pm 6.65$ vs. $81.59 \pm 7.55 \%$; Fig. 2C). Under the experimental condition of 1-min duration, $20 \% \mathrm{DC}$ and $2 \mathrm{w} / \mathrm{cm}^{2}$ intensity, the increase in the ratio of MBs to cells (20:1, 50:1 and 80:1) resulted in an increase in cell mortality. The viability of the cell was lowest in the 80:1 group $(66.40 \pm 7.64 \%)$, and no significant difference was identified between the 20:1 and the 50:1 MB concentration groups $(84.72 \pm 6.65$ vs. $83.56 \pm 4.37 \%$;
Fig. 2D). Under the optimal US parameters (frequency, $1 \mathrm{MHz}$; pulse recurrent frequency, $100 \mathrm{~Hz}$; intensity, $\leq 2 \mathrm{w} / \mathrm{cm}^{2}$; duration, $1 \mathrm{~min}$; pulse wave, 20 and $50 \% \mathrm{DC}$; and ratio of MBs to cells, 20:1 and 50:1), the cell viability was $>80 \%$, and the cells were equally distributed with no significant cell damage. The optimal parameters were used in the following experiments.

Gene transfer by pEGFP alone, $p E G F P+U S, P E I / p E G F P$ and PEI/pEGFP + US. Compared with the group subjected to pEGFP alone $(0.63 \pm 0.18 \%)$, the pEGFP + US group $(1.09 \pm 0.34 \%)$ exhibited a weak but non-significant tendency to improve transgene expression. Compared with the pEGFP alone and pEGFP + US groups, the PEI/pEGFP (7.31 $1.06 \%)$ and PEI/pEGFP + US $(7.46 \pm 1.04 \%)$ groups exhibited a significantly higher expression of the EGFP-positive cells. However, no significant improvement was observed in the transgene expression between the PEI/pEGFP + US and PEI/pEGFP group.

UTMD-mediated PEI/pEGFP transfection. To optimize the conditions of the UTMD-mediated PEI/pEGFP transfection of the ARPE-19 cells, different parameters were examined. 
A

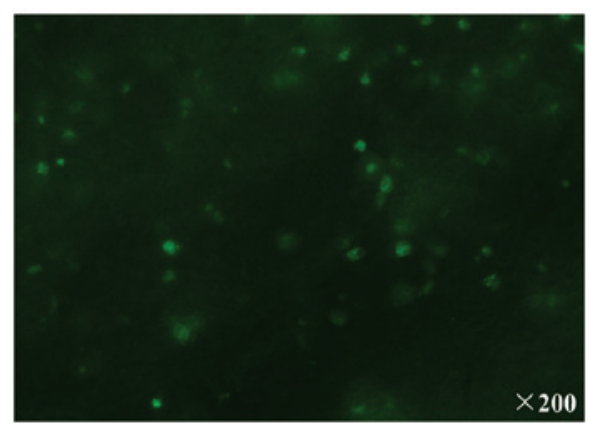

C

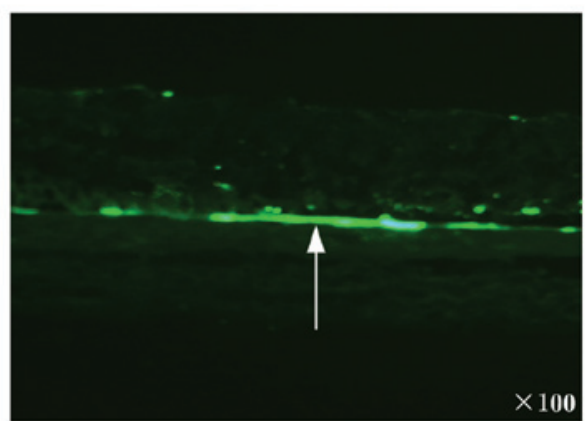

B

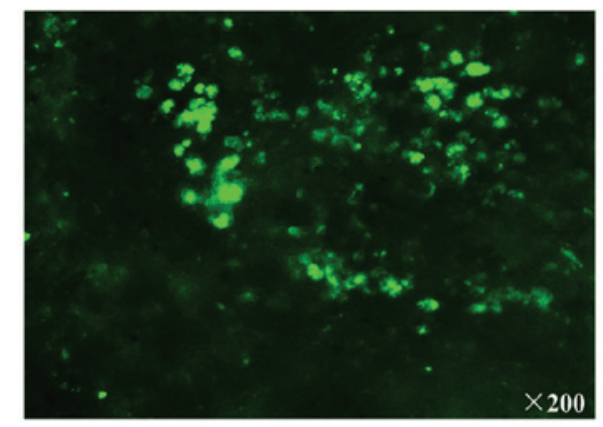

D

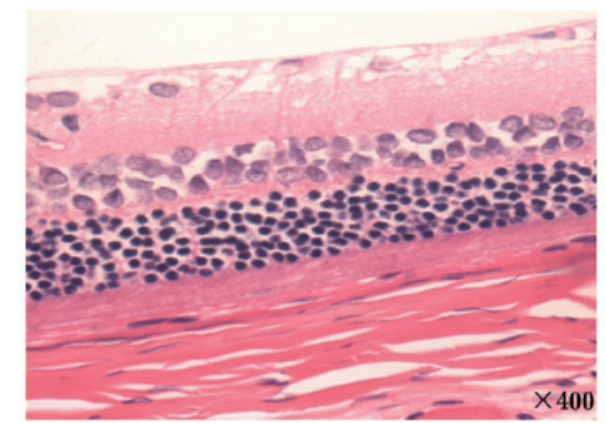

Figure 4. Tissue evaluation, frozen sections and histology of optic cups. (A) Star-like EGFP-positive cells (weak positive) were observed in the rats from each group. (B) In the group treated with PEI/pEGFP+UTMD, pEGFP-positive cells were patchy in distribution. (C) Frozen sections of optic cups exhibited pEGFP-positive cells mainly distributed in the layer of retina (arrow). (D) Hematoxylin and eosin staining revealed that no marked tissue damage was observed following ultrasound-targeted microbubble destruction-mediated PEI/pEGFP transfection. pEGFP, enhanced green fluorescent protein plasmid; PEI, polyethylenimine; US, ultrasound; UTMD, ultrasound-targeted microbubble destruction.

The pulse waves of 20 and 50\% DC were examined for $1 \mathrm{~min}$ duration, $1 \mathrm{w} / \mathrm{cm}^{2}$ intensity, and the ratio of the MBs to the cells was at 50:1. The EGFP-positive ratio was significantly higher in the $50 \%$ DC group $(12.48 \pm 1.01 \%)$ than that in the $20 \%$ DC group $(9.13 \pm 0.68 \%)$ (Fig. $3 \mathrm{~A})$. The parameter of $50 \%$ $\mathrm{DC}$ was selected for the following experiments.

The US intensities of 1 and $2 \mathrm{w} / \mathrm{cm}^{2}$ were examined under the condition of $50 \% \mathrm{DC}, 1-\mathrm{min}$ duration and the ratio of the MBs to the cells 50:1. No significant difference was identified in the EGFP-positive ratio between the 1 and $2 \mathrm{w} / \mathrm{cm}^{2}$ groups $(12.48 \pm 1.01 \%$ vs. $13.45 \pm 0.89 \%$, respectively; Fig. 3B). In consideration of the effect of the UTMD on cell viability, $1 \mathrm{w} / \mathrm{cm}^{2}$ was selected as the optimal parameter.

Under the condition of $50 \%$ DC, 1-min duration and $1 \mathrm{w} / \mathrm{cm}^{2}$ intensity, the optimal ratio of the MBs to the cells (20:1 and 50:1) was selected. The EGFP-positive ratio was the highest in cells treated by US with the ratio of the MBs to the cells at 50:1 (Fig. 3C). Thus, a ratio of the MBs to the cells at 50:1 was determined to be appropriate.

In vivo study. Star-like EGFP-positive cells (weak positive; Fig. 4A) were observed in the rats from each groups. Negative expression was observed only in the rats of the PEI/pEGFP group (Table I). Diffuse EGFP-positive cells were observed (strong positive; Fig. 4B) in 7 of the 12 rats in the group treated with PEI/pEGFP complexes with UTMD (Table I). The difference between the experimental group and the control group was significant $(\mathrm{P}=0.0038)$. The frozen sections of the optic cups revealed green fluorescence, predominantly distributed in the retina (Fig. 4C). The histological analysis revealed that no marked tissue damage was present in the UTMD group following transfection (Fig. 4D).

\section{Discussion}

With advances in the preparation technology of MBs and the innovations in US imaging, contrast-enhanced US is no longer confined to the detection of tissue perfusion, but has gradually extended to specific US molecular imaging and targeted therapy. MBs as cavitation nuclei are able to volumetrically expand and contract in response to the compression and rarefaction phases of US waves (16). When the acoustic pressure reaches a certain threshold, MBs may collapse violently and cause a series of biological effects. The physical responses of MBs are able to mechanically perturb the integrity of blood vessel walls and cell membranes, thus increasing their permeability to therapeutic agents (16). In recent years, numerous proof-of-principle studies have indicated that the combination of low-intensity US and MBs allows direct DNA transfer into the cytosol through small pores in the cells caused by cavitation effects, and enhances gene transfection in vitro and in vivo (17-19).

Non-viral vectors for gene delivery offer a host of potential advantages over viruses. However, compared with viral systems, the majority of non-viral vectors are less efficient with hard-to-transfect cell types (20). Multiple alternative non-viral gene delivery approaches have been reported for the in vitro transfection of RPE cells, but the results were unsatisfactory. A previous study was designed to evaluate the transfection capacity of solid lipid nanoparticles in the human RPE 
established cell lines. The results demonstrated that the transfection efficacy was low, and only $2.5 \%$ EGFP positive cells were observed at $72 \mathrm{~h}$ after transfection (21). Urtti et al (22) reported that the transfection efficiencies of primary RPE cells were $<1 \%$ for lipofectin, $<5 \%$ for degraded dendrimers, and between 1 and $3 \%$ when using DOTAP/DOGS. The low and unsatisfactory transfection efficiency of RPE cells in vitro indicates a requirement for more efficient delivery systems to augment the transfection efficiency. The present study was designed to determine whether the combination of UTMD with PEI was able to enhance the gene transfection of the RPE cells in vitro and in vivo.

To establish the optimal conditions of UTMD-mediated gene transfer to human RPE cells, various US conditions were examined. The US intensity, exposure duration, DC and MB concentration were examined. The present results revealed that the viability of the cells reduced with an increase in the exposure intensity, duration, DC and the ratio of the MBs to cells. Considering cell viability and gene transfer efficiency, the optimal UTMD condition was as follows: US intensity, $1 \mathrm{w} / \mathrm{cm}^{2}$; exposure duration, $1 \mathrm{~min}$; DC, $50 \%$; and ratio of the MBs to cells, 50:1.

In the present study, PEI-EGFP complexes with or without US were transfected into the human RPE cells with a $7.45 \pm 1.25$ and $7.46 \pm 1.04 \%$ transfection efficacy, respectively. Similarly to the present study, Sunshine et al (23) demonstrated that branched $25 \mathrm{kDa}$ PEI transfected ARPE-19 cells with an $8 \pm 1 \%$ transfection efficacy. A number of studies have revealed that ultrasound-mediated transfection (USMT) is able to enhance gene transfer efficiency, but the present findings indicated that US alone without MBs was not able to increase the transfer efficiency. It is possible that a higher US intensity is required for the transient pore formation in human RPE cells, but this may decrease the viability of cells.

UTMD may facilitate targeted gene transfection, thus significantly enhance gene transfection in vitro. Under optimal conditions, UTMD-mediated gene transfection was $12.48 \pm 1.01 \%$, which was significantly greater than other groups without causing any apparent adverse effects. The transfection efficiencies of UTMD and PEI were enhanced 19.8-fold compared with transfection of an EGFP plasmid alone. The results of the present in vitro study revealed that UTMD was able to significantly enhance PEI-mediated gene expression in RPE cells.

In the area of ophthalmology, investigations into UTMD-mediated gene delivery have predominantly focused on the cornea and retina. Sonoda et al (11) performed a study to estimate the practical efficacy and safety of US with MB-mediated gene delivery to the cornea. The results revealed that US alone exerted no significant enhancement in gene transfer in the in vivo study. However, US with MBs is able to markedly increase GFP gene transfer in vivo and in vitro without causing any apparent side effects, and the transfer efficiency was significantly higher than that with US or naked plasmid alone. Li et al (12) reported that UTMD was able to enhance rAAV2 transfection efficiency in human RPE cells in vitro and in rat retina in vivo, but USMT and MBs alone did not affect the transfection efficiency of the retina in vivo. In the present study, no EGFP-positive cells (negative) and star-like EGFP-positive cells (weak positive) were observed in the rats treated with PEI-pEGFP alone. Diffuse EGFP-positive cells were observed (strong positive) in 7 of the 12 eyes in the UTMD group, and the histological analysis demonstrated that no marked tissue damage was present in the UTMD group following transfection.

Multiple administrative routes are currently used to deliver bio-active materials to the eye. If the retina is the target, topical, systemic and periocular approaches are limited by the blood-retinal barrier (BRB) and other ocular barriers. In the present study, subretinal injection was selected, which is able to provide direct access to the retinal tissues. Due to the risk of retinal detachment and hemorrhage, this invasive method is not the first choice for the treatment of diseases of the eye clinically. Therefore the development of non-invasive and efficient methods, which are able to bypass the BRB and enhance the delivery of therapeutic materials to the retina is important. Park et al (24) demonstrated that the appropriate intensity of focused US combined with MBs are able to induce a temporary and reversible disruption of the BRB in rats, and the barrier was found to be restored within a few hours after sonication. In their study, no significant retinal damage was identified in the histology at the two lower acoustic pressure amplitudes test. US with MBs may offer a noninvasive, localized and repeatable means to reversibly disrupt the BRB for the delivery of ocular substances, which requires further investigation.

As the SonoVue membranes and DNA bear a negative charge (25), the binding of the SonoVue MB and the plasmid DNA may be weak and transient. PEI, as one of the most effective cationic gene vectors, is able to condense DNA into compact particles via its electrostatic interaction. The PEI/DNA complexes may be adsorbed to the surface of MBs through electrostatic interaction. They may form a DNA/PEI/SonoVue complex. The complex could be released when targeted with US irradiation, and enhance the transfection efficiency without off-target effects. In the present study, the transfection efficiency was enhanced following treatment. With an increase in the N/P ratio, the viability of the cells may be reduced (26). In the present study, a relatively low N/P ratio $(\mathrm{N} / \mathrm{P}=8)$ was selected, and the results revealed that this $\mathrm{N} / \mathrm{P}$ ratio was able to increase the transfection efficiency effectively in vitro and in vivo. However, the efficiency of different $\mathrm{N} / \mathrm{P}$ ratios should be analyzed in further investigations.

In the present study, it was demonstrated that the combination of UTMD and PEI was able to significantly enhance the gene expression of plasmid DNA in human RPE cells in vitro and in SD rat retina in vivo without any apparent tissue damage. UTMD provided a new method for the systemic administration of the non-viral vectors. Although this method was highly dependent upon the acoustic conditions and the MB concentration, its simplicity and non-invasiveness could provide a safe and effective non-viral gene delivery system for the gene transfer of certain inherited and acquired diseases of the retina.

\section{Acknowledgements}

The present study was supported by grants from the Natural Science Foundation of Shanghai Science Commission (no. 11ZR1421100), the Scientific Effort Project of Shanghai 
Science and Technology committee (no. 1441968200) and the Natural Science Foundation of China (no. 81200700).

\section{References}

1. Surace EM and Auricchio A: Versatility of AAV vectors for retinal gene transfer. Vision Res 48: 353-359, 2008.

2. Zhang L, Li X, Zhao M, He P, Yu W, Dong J et al: Antisense oligonucleotide targeting c-fos mRNA limits retinal pigment epithelia cell proliferation; a key step in the progres- sion of proliferative vitreo-retinopathy. Exp Eye Res 83: 1405-1411, 2006.

3. Lehrman S: Virus treatment questioned after gene therapy death. Nature 401: 517-518, 1999.

4. Liu Q and Muruve DA: Molecular basis of the inflammatory response to adenovirus vectors. Gene Ther 10: 935-940, 2003.

5. Sun JY, Anand-Jawa V, Chatterjee S and Wong KK: Immune responses to adeno-associated virus and its recombinant vectors. Gene Ther 10: 964-976, 2003.

6. Glover DJ, Lipps HJ and Jans DA: Towards safe, non-viral therapeutic gene expression in humans. Nat Rev Genet 6: 299-310, 2005 .

7. Park HJ, Yang F and Cho SW: Nonviral delivery of genetic medicine for therapeutic angiogenesis. Adv Drug Deliv Rev 64: 40-52, 2012.

8. Zhigang W, Zhiyu L, Haitao R, et al: Ultrasound-mediated microbubble destruction enhances VEGF gene delivery to the infarcted myocardium in rats. Clin Imaging 28: 395-398, 2004.

9. Zhang Q, Wang Z, Ran H, et al: Enhanced gene delivery into skeletal muscles with ultrasound and microbubble techniques. Acad Radiol 13: 363-367, 2006.

10. Ren JL, Wang ZG, Zhang Y, et al: Transfection efficiency of TDL compound in HUVEC enhanced by ultrasound-targeted microbubble destruction. Ultrasound Med Biol 34: 1857-1867, 2008.

11. Sonoda S, Tachibana K, Uchino E, et al: Gene transfer to corneal epithelium and keratocytes mediated by ultrasound with microbubbles. Invest Ophthalmol Vis Sci 47: 558-564, 2006.

12. Li HL, Zheng XZ, Wang HP, Li F, Wu Y and Du LF: Ultrasound-targeted microbubble destruction enhances AAV-mediated gene transfection in human RPE cells in vitro and rat retina in vivo. Gene Ther 16: 1146-1153, 2009.

13. Neu M, Fischer D and Kissel T: Recent advances in rational gene transfer vector design based on poly (ethylene imine) and its derivatives. J Gene Med 7: 992-1009, 2005.
14. Weerasinghe P, Li Y, Guan Y, Zhang R, Tweardy DJ and Jing N: T40214/PEI complex: a potent therapeutics for prostate cancer that targets STAT3 signaling. Prostate 68: 1430-1442, 2008.

15. Hu YZ, Zhu JA, Jiang YG and Hu B: Ultrasound microbubble contrast agents: application to therapy for peripheral vascular disease. Adv Ther 26: 425-434, 2009.

16. Qin S, Caskey CF and Ferrara KW: Ultrasound contrast microbubbles in imaging and therapy: physical principles and engineering. Phys Med Biol 54: R27-R57, 2009.

17. Chen YC, Jiang LP, Liu NX, Wang ZH, Hong K and Zhang QP: P85, Optison microbubbles and ultrasound cooperate in mediating plasmid DNA transfection in mouse skeletal muscles in vivo. Ultrason Sonochem 18: 513-519, 2011.

18. Chen S, Shimoda M, Wang MY, et al: Regeneration of pancreatic islets in vivo by ultrasound-targeted gene therapy. Gene Ther 17: 1411-1420, 2010.

19. Koike H, Tomita N, Azuma H, et al: An efficient gene transfer method mediated by ultrasound and microbubbles into the kidney. J Gene Med 7: 108-116, 2005.

20. Sunshine JC, Bishop CJ and Green JJ: Advances in polymeric and inorganic vectors for nonviral nucleic acid delivery. Ther Deliv 2: 493-521, 2011.

21. del Pozo-Rodríguez A, Delgado D, Solinís MA, Gascón AR and Pedraz JL: Solid lipid nanoparticles for retinal gene therapy: transfection and intracellular trafficking in RPE cells. Int J Pharm 360: 177-183, 2008.

22. Urtti A, Polansky J, Lui GM and Szoka FC: Gene delivery and expression in human retinal pigment epithelial cells: effects of synthetic carriers, serum, extracellular matrix and viral promoters. J Drug Target 7: 413-421, 2000.

23. Sunshine JC, Sunshine SB, Bhutto I, Handa JT and Green JJ: Poly ( $\beta$-amino ester)-nanoparticle mediated transfection of retinal pigment epithelial cells in vitro and in vivo. PLoS One 7: e37543, 2012

24. Park J, Zhang Y, Vykhodtseva N, Akula JD and McDannold NJ: Targeted and reversible blood-retinal barrier disruption via focused ultrasound and microbubbles. PLoS One 7: e42754, 2012.

25. Yanagisawa K, Moriyasu F, Miyahara T, Yuki M and Iijima H: Phagocytosis of ultrasound contrast agent microbubbles by Kupffer cells. Ultrasound Med Biol 33: 318-325, 2007.

26. KircheisR,WightmanL,Schreiber A, etal:Polyethylenimine/DNA complexes shielded by transferrin target gene expression to tumors after systemic application. Gene Ther 8: 28-40, 2001. 